\title{
Optical monitoring of extragalactic sources for linking the ICRF and the future Gaia celestial reference frame
}

\section{Variability of ICRF sources ${ }^{\star}$}

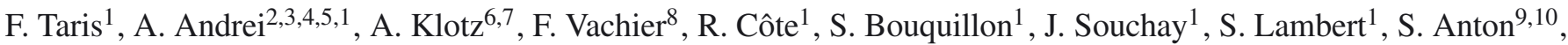 \\ G. Bourda ${ }^{11,12}$, and D. Coward ${ }^{13}$
}

1 Observatoire de Paris - SYRTE, CNRS/UMR 8630 \& Université Pierre et Marie Curie, 75005 Paris, France e-mail: Francois.Taris@obspm.fr

2 Observatório Nacional/MCT, 20921-400 Rio de Janeiro, Brazil

3 Obervatorio do Valongo, UFRJ, Brazil

4 Osservatorio Astronomico di Torino, INAF, 10025 Pino Torinese, Italy

5 Shangai Astronomical Observatory, CAS, 200030 Shanghai, PR China

${ }^{6}$ Université de Toulouse, UPS/OMP, IRAP, 31400 Toulouse, France

7 CNRS, IRAP, 14 avenue Édouard Belin, 31400 Toulouse, France

8 Observatoire de Paris - IMCCE, 75005 Paris, France

9 CICGE, Faculdade de Ciências da Universidade do Porto, 4150-564 Porto, Portugal

10 SIM, Faculdade de Ciências da Universidade de Lisboa, 1749-016 Lisbon, Portugal

11 Université de Bordeaux, OASU, 2 rue de l'Observatoire, BP 89, 33271 Floirac Cedex, France

12 CNRS, UMR 5804, LAB, 2 rue de l'Observatoire, BP 89, 33271 Floirac Cedex, France

13 School of Physics, University of Western Australia, Crawley WA 6009, Australia

Received 25 May 2012 / Accepted 3 December 2012

\section{ABSTRACT}

\begin{abstract}
Context. The astrometric mission Gaia of the European Space Agency is scheduled to be launched in 2013. It will provide an astrometric catalog of 500000 extragalactic sources that could be the basis of a new optical reference frame after the HIPPARCOS satellite one. On the other hand, the current International Celestial Reference Frame (ICRF) is based on observations of extragalactic sources at radio wavelength. The astrometric coordinates of sources in these two reference systems will have roughly the same uncertainty. It is then mandatory to observe a set of common targets at both optical and radio wavelengths to link the ICRF with what could be called the Gaia Celestial Reference Frame (GCRF).

Aims. The goal of this work is to observe a first set of 70 extragalactic sources at optical wavelengths that could achieve the link with the ICRF. Variations in the light curves of these targets are connected with astrophysical processes that could produce displacements of the optical photocenter. Such displacements, if they exist, are critical in the framework of the link of reference systems.

Methods. Four telescopes were used to observe the targets at optical wavelengths. Two of them are located in France, one in Chile, and the last one in Australia. First observations were carried out during one year and a half in the $R$ and $V$ bands. A new method of characterizing the compactness of the targets was applied to the images obtained.

Results. This paper presents results for the optical monitoring of extragalactic sources suitable for linking reference systems. We show that a large number of targets in our set are variable at the two observational wavelengths. A short presentation of each object is given, along with some references to earlier photometric studies. A morphological index is defined and applied to the 5000 images obtained during the observation campaign.

Conclusions. This work fits into a more general project of astrophotometric and astrophysical studies of extragalactic radiosources in the framework of the reference systems. It brings to the astrometric community some information at optical wavelengths about a set of targets that could be used for the link between the radio ICRF and the future GCRF.
\end{abstract}

Key words. reference systems - astrometry - quasars: general

\section{Introduction}

The unified model for active galactic nuclei (AGN) is a well known and already accepted model for a set of objects that were previously thought to be different. It postulates that the appearance of AGN depend strongly on its orientation to the observer's line of sight in addition to its intrinsic physical properties. The unified model is described by Antonucci (1993), Urry \& Padovani (1995), and others. The AGN phenomenon accounts

* Table 4 is only available in electronic form at the CDS via anonymous ftp to cdsarc.u-strasbg. fr (130.79.128.5) or via http://cdsarc.u-strasbg.fr/viz-bin/qcat?]/A+A/552/A98 for different objects, such as blazars, radio-loud or radio-quiet quasars, Seyfert galaxies, and narrow or broad line radio galaxies. They are all connected by various common physical components, such as a black hole (possibly a binary one), an accretion disk, broad and narrow line regions, an obscuring torus, and a jet.

For a very long time, AGN have been presented as unchanging point-like objects at optical wavelengths because they were extragalactic sources at cosmological distances. With that point of view they can be used to realize a quasi-inertial celestial reference frame. Nevertheless, it has been pointed out that these objects are not point-like sources at radio wavelengths and that a structure index can be computed (Fey \& Charlot 1997). The intrinsic and extended radio structure of the extragalactic radio 
sources, as well as its evolution, is one of the limiting factors for defining the International Reference Frame (ICRF). Sources are classified either as defining sources (which were used to set the axes of the frame), candidate sources (less observed), or other sources. Significant proper motions due to intrinsic changes in the source structure have been pointed out by Fey et al. (1997) for the case of the source $4 \mathrm{C} 39.25$. As a matter of fact, the position instabilities of the radio center makes some sources inadequate for being used as defining sources during specific periods of time. For this reason, the monitoring of the ICRF sources is a task that is currently in progress in the framework of the IERS-PC ${ }^{1}$ (International Earth Rotation Service-Product Center) and the IVS-OPAR ${ }^{2}$ (International VLBI Service-Observatoire de Paris).

Physical processes may have both an optical and a radio signature. Physical processes with a signature in the optical domain could be located for example in the accretion disk (Li \& Cao 2008; Shalyapin et al. 2002), in the jet (Bahcall et al. 1995; Cheung 2003), or near the black hole (Lobanov \& Roland 2005). Even though it is an extrinsic phenomenon, the long-term optical variability in AGN could be due to gravitational microlensing (Hawkins et al. 2003).

Regardless of the physical process at the origin of an optical magnitude variation, the size of the emission region and the distance of the AGN is especially important for linking the reference systems. This is particularly true in the scope of the Gaia catalog where questions about structural modifications in the optical domain could arise and consequently be a limiting factor for accuracy. A preliminary work has recently been published about the astrophotometric variability of the CFHT-LS Deep 2 QSOs (Taris et al. 2011).

Another potential limiting factor is the presence of a host galaxy around the nucleus of the object. In this case determination of the centroid could be affected by the shape of this surrounding galaxy. This determination could also depend on the observation wavelength.

The goal of this work is to observe a first set of 70 extragalactic sources at optical wavelengths that could achieve the link with the ICRF. Variations in the light curves of these targets are associated with astrophysical processes that could produce displacements of the optical photocenter. These displacements, if they exist, would be critical in linking the reference systems.

In the next section we present the telescopes used and our data analysis methods. Then we present the main characteristics of the reference systems discussed in this work. Notes on individual targets and light curves obtained follow before a discussion about the morphological index of the observed targets. The conclusion is devoted to the works in progress to complete these first observational results.

\section{Telescopes, observations, and data analysis}

The observations were carried out with four telescopes located in France, Chile, and Australia. This section presents these telescopes and the method used to achieve the photometric reduction of the data.

\section{1. $T 120$}

The $1.2 \mathrm{~m}$ telescope (Observatoire de Haute Provence ${ }^{3}$, IAU code 511) was used for 17 nights from March 2010 to

\footnotetext{
1 See http://hpiers.obspm.fr/icrs-pc/

2 See http://ivsopar.obspm.fr

3 See http://www.obs-hp.fr
}

February 2011 with a $1024 \times 1024$ CCD camera with a pixel scale of $0.69 \mathrm{arcsec} / \mathrm{px}$ giving a field of view of $10 \mathrm{sq}$. arcmin. The seeing during the observations was relatively poor, with a mean value of 3.4 arcsec. The filters used were the $V$ and $R$ Cousins filters.

\subsection{Tarots}

TAROT (Klotz et al. 2008) stands for Télescope à Action Rapide pour l'Observation des phénomènes Transitoires. It is composed of two identical robotic telescopes, one of them located at Observatoire de la Côte d'Azur ${ }^{4}$ ) and the other one at ESO La Silla. TAROT are $25 \mathrm{~cm} F / D=3.4$ telescopes covering $1.86 \times 1.86 \mathrm{deg}^{2}$ field of view on the Andor CCD cameras (Marconi 4240 back illuminated). Spatial sampling is $3.3 \mathrm{arcsec} /$ pix. Six filters are available: $B V R I$, a clear filter, and a 2.7 density coupled to $V$ (for Moon and planets). The detection limit is about $V=17$ in 1-min exposure time. These two telescopes have been in use since February 2011.

\subsection{Zadko}

$\mathrm{Zadko}^{5}$ is a $1-\mathrm{m}$ robotic telescope funded by a donation from James Zadko to the University of Western Australia (UWA). It is a $1-\mathrm{m} f / 4$ Cassegrain telescope located in the state of Western Australia about $67 \mathrm{~km}$ north of Perth. It is equipped with an Andor $2048 \times 2048$ back-illuminated CCD. The field of view is $23.5 \operatorname{arcmin}^{2}$ and the filters used for this study are $g$ and $r$ Gunn filters. Zadko and TAROT are part of the same robotic telescope network for space-debris identification and tracking (Laas-Bourez et al. 2010). Currently, five robotic observatories are linked to a central observatory coordinator CADOR (Coordination et Analyse des Données des Observatoires Robotiques).

\subsection{Observations and data analysis}

The OHP images were reduced with the Image Reduction and Analysis Facility (IRAF ${ }^{6}$ ). The images were bias-substracted and flat-fielded using the imcombine and imarith routines. A first astrometric reduction used the ccxymath routine because the header of the fits files does not contain the required $\mathrm{WCS}^{7}$ keywords. Photometric reduction used customized software (for details about the protocol see Taris et al. 2011) after the detection of the stars by Sextractor software ${ }^{8}$ (Bertin \& Arnouts 1996). The magnitude of these stars were compared to the known magnitudes of the stars in the GSC2.3 catalog (Lasker et al. 2008). After a $3 \sigma$ rejection of the outliers, the formal uncertainty of the photometric calibration is roughly 0.2 mag in both $R$ and $V$.

The photometric reduction of the TAROT and Zadko telescopes is described by Damerdji et al. (2007). It is based on a measurement flux by the Sextractor software. The precision of the photometry is 0.05 mag relative to the same reference star chosen close to the target. The accuracy is only 0.4 mag, since it

\footnotetext{
4 See http://tarot.obs-hp.fr/tarot

5 See http://www.zt. science.uwa.edu.au

6 IRAF is distributed by the National Optical Astronomy Observatories, which are operated by the Association of Universities for Research in Astronomy, Inc., under cooperative agreement with the National Science Foundation.

7 See for example

http://www . atnf. csiro.au/people/mcalabre/WCS/

8 See http://www . astromatic.net/software/sextractor
} 
is limited by the photometric calibration of the USNO A2 cata$\log$. However, because this study is concerned with photometric time variation, we retain the value of 0.05 mag as the uncertainty of a series of measures obtained by a given telescope.

\section{Reference frames}

The current conventional realization of the ICRS is the second version of the ICRF called ICRF2. In the optical domain, the HIPPARCos catalog is the current international conventional realization, but in the future (around 2020) the Gaia optical catalog should lead to an optical update of the ICRF. The link between these reference frames, in the radio and in the optical domains, is of course significant. In this section, we present all of these fundamental concepts in a didactic way.

\subsection{ICRF2}

At the 2009 XXVIIth IAU General Assembly in Rio, Brazil, the astronomical community adopted the second release of the International Celestial Reference Frame named ICRF2 (Fey et al. 2009) as the new fundamental astrometric materialization of the ICRS. From 1 January 2010, the ICRF2 replaces the ICRF (Ma et al. 1998) and its most recent extension, the ICRF-Ext2 (Fey et al. 2004).

The construction of the ICRF2 used almost 30 years of geodetic very long baseline interferometry (VLBI) observations at $3.6 \mathrm{~cm}$ and $13 \mathrm{~cm}$ wavelengths. The ICRF2 catalog contains positions of 3414 compact radio sources. The formal errors $\sigma$ in source coordinates increased according to $\left((1.5 \sigma)^{2}+\sigma_{0}^{2}\right)^{1 / 2}$ where $\sigma_{0}$ is a noise floor set to $40 \mu$ as. The median error in the position of sources observed in more than two sessions is $175 \mu$ as. The frame axes are defined by the coordinates of 295 "defining" sources with a stability of $\sim 10 \mu$ as. The defining sources were chosen on the basis of their high positional stability and low structure index. A subset of 138 defining sources was used to align the ICRF2 catalog onto the ICRF.

The ICRF2 currently represents the most accurate realization of the celestial system with respect to which the position of any object in the celestial sphere should be measured. We note that the ICRF is epochless and independent of the dynamical frame (ecliptic) and reference point (equinox), but is consistent with previous realizations of the ICRS, including the FK5 J2000.0 optical system.

\subsection{The future Gaia celestial reference frame}

The European astrometric space mission Gaia will be launched in 2013. It will provide positions and proper motions of around one billion stars and about 500000 QSOs with unprecedented uncertainty between the 6th and the 20th magnitudes (Lindegren 2009). The predicted accuracy is a few hundred $\mu$ as at the 20th magnitude. To prepare the future Gaia extragalactic reference frame, a clean sample of at least 10000 QSOs must be implemented (Gaia initial quasar list). This work is being performed in the framework of the Gaia workpackage GWP-S-335-13000 (led by A. Andrei) with the aim of offering an initial QSO catalog.

Two catalogs are at the basis of this work, the LQAC (Souchay et al. 2009) and the LQRF (Andrei et al. 2009). The LQAC contains 113666 quasars. It is a compilation of the 12 largest quasar catalogs (4 from radio VLBI programs,
8 from optical surveys). Information about $u, b, v, g, r, i, z, J$, $K$ photometry, as well as redshift and radio fluxes at $1.4 \mathrm{GHz}$, $2.3 \mathrm{GHz}, 5.0 \mathrm{GHz}, 8.4 \mathrm{GHz}$, and $24 \mathrm{GHz}$ are given when available. A small fraction of the remaining objects that are not present in the 12 catalogs, but included in the Véron-Cetty \& Véron quasar catalogs (Véron-Cetty \& Véron 2006), have been added to the compilation. The first version of the LQAC has been recently superseded by a second release (Souchay et al. 2012).

The LQRF contains 100165 quasars that are represented well across the sky, from $-83.5^{\circ}$ to $+88.5^{\circ}$ in declination. The average distance to the nearest neighbor QSO is 10.4 arcmin, and it is smaller than 10 arcmin for $78 \%$ of the sources. The global alignment with the ICRF is 1.5 mas, and the individual position accuracies are represented by a Poisson distribution peaking at 139 mas in right ascension and 130 mas in declination. The LQRF contains equatorial coordinates at epoch J2000.0, and is complemented by redshift and photometric information from the LQAC.

\subsection{The link between ICRF2 and the Gaia extragalactic reference frame}

Relating the ICRF2 to the Gaia extragalactic reference frame will be a very important task in the near future, and some works are currently underway to achieve this goal. An investigation of the correlation between the long-term optical variability and the photocenter motion of QSOs is being pursued at the ESO Max Planck $2.2 \mathrm{~m}$ telescope in Chile (Andrei et al. 2008). A sample of quasars has been selected in terms of their large amplitude and long-term optical variability. The observations are typically performed every two months. The analysis procedure is completely differential: the quasar positions and brightness are determined starting from a set of selected stars for which the average relative distances and magnitudes remain constant. The preliminary results for four objects strongly support the hypothesis of a relationship between astrometric and photometric variability. If verified on a larger sample, this relationship would mean that large photometric variations would hamper a given quasar from realizing as stable an extragalactic reference frame as the one provided by the GAIA mission (Anton et al. 2012).

Bourda et al. (2008) evaluated the suitability of the current individual ICRF-Ext2 (the ICRF catalog that preceded the ICRF2) extragalactic radio sources for the alignment with the future Gaia frame. They identify 243 candidates among the ICRF-Ext2 sources with an optical counterpart that is brighter than the apparent magnitude $V=18$. Among these 243 candidates, only 70 have data of excellent or good astrometric quality (i.e., an $X$-band structure index value of either 1 or 2 ) for determining the Gaia link with the highest accuracy. Nevertheless, this index value is perhaps not well suited to determining the best sources in the optical domain in that several sources given by these authors are not point-like sources (for example, NGC 3031 or Messier 81, MARK 421, NGC 4374, or Messier 84). The goal of our current work is to observe this first set of 70 extragalactic sources at optical wavelengths. Magnitude variations are especially important because they indicate the maximum size of the emission regions, and since the distance is known, the maximum apparent amplitude of the random walk of the photocenter.

\section{Notes on individual targets and light curves}

The following notes were collected from the SIMBAD (Set of Identification Measurements and Bibliography for Astronomical 
Data) data base ${ }^{9}$ before the 29th July 2011. They present an overview of what is known about the targets in the optical domain both for the magnitude variability and for the morphology. A summary of the following notes, in terms of variability and morphology, is presented in the Table 2. The original light curves that we obtained with the telescopes mentioned above are the subject of the next section. They present our contribution to the study of the targets. The journal of our observations is given in Table 3.

\subsection{Notes on individual targets}

Among the 70 targets of the initial set, three of them, M 81, M 84, and Mrk 421, are very well known and obviously not QSOs at optical wavelengths. Even though they are point-like objects at radio wavelengths, the choice was made to remove them from the initial set, hence to not observe them in the optical domain.

Among the 67 remaining targets, 28 have been observed elsewhere for their structure and it has been firmly established that 14 targets have and 14 do not have a prominent host galaxy in the optical bands. Two targets (QSO B0422+004 and QSO B0754+100) seem to have observations that are about the existence of a host galaxy, and for eight of them (QSO B1144+402, QSO B1508-055, QSO B1725+044, QSO B1954-388, QSO B2059+034, QSO B2126-158, QSO B2143-156, and QSO B2227-088), the morphology appears to be uncertain. For 17 targets $(25 \%$ of the initial list) high-resolution images in the HST archive ${ }^{10}$ are currently under investigation in the search for the host galaxies. For 22 targets (33\% of the initial list), dedicated high-resolution observations are currently planned at ESO (MPG2.2 Telescope), in Hawaii (3.6 m Canada France Hawaii Telescope), and in Chile (SOAR Telescope) to search for the host galaxies. This work on morphology will be the subject of a forthcoming paper.

Some targets (10 among the 67) were difficult to observe with our small telescopes due to their faintness. One other is very close to a bright star. Consequently, these 11 targets will remain difficult to observe with other small telescopes.

Among the 67 targets concerned, 23 of them have been observed for a long time. and some light curves have been found in refereed papers. For 37 targets (55\% of the initial list), the light curves presented in this work bring new information. Among these 37 targets, five (QSO B1508-055, QSO B1725+044, QSO B1954-388, QSO B2126-158, QSO B2227-088) only have one measure of magnitude reported in the literature, and two of them (QSO B0955+326 and QSO B1749+096) have intranight variability. Seven of the 67 targets remain unobserved in the framework of this work.

QSO B0007+106

This object is classified as a Seyfert I galaxy in SIMBAD, and it was soon recognized as a nonstellar source by Zwicky (Arp 1968). More recent studies about the morphology of the target at optical and near IR wavelengths are available (Slavcheva-Mihova \& Mihov 2011; Surace et al. 2001). The variability of the target has been observed in the optical (Smith \& Nair 1995) and in near IR (Glass 2004).

QSO B0010+405

This object is classified as a Seyfert I galaxy in SIMBAD. Only one paper (Vigotti et al. 1989) mentions this target as nonstellar

\footnotetext{
9 See http://simbad.u-starsbg.fr/simbad

${ }^{10}$ http://archive.stsci.edu/
}

(compact galaxy). No light curve in the optical domain has been found in the bibliography.

\section{QSO B0048-097}

This object is classified as a BL Lac in SIMBAD. The magnitude variation is very well known and has been followed for more than 40 years (Fan \& Lin 2000a; Pica et al. 1988). With highresolution NTT images, this target shows a close companion about 2.5 arcsec east of the nucleus. The nucleus is unresolved, and its radial brightness profile matches that of a point source PSF perfectly (Falomo 1996).

\section{QSO B0109+224}

This radio source is classified as a BL Lac. It exhibits significant variability in optical bands, and the host galaxy was not detected in the high-resolution images obtained at the NTT and NOT (Sbarufetti et al. 2006).

\section{QSO B0202+319}

No information on variability and morphology was found for this target in SIMBAD.

\section{QSO B0208-512}

QSO B0208-512 is classified as a BL Lac. The host galaxy has been marginally resolved in the frame of a high-resolution near-IR $H$-band imaging survey (Kotilainen et al. 1998a). No information was found about the morphology and magnitude variation in the optical domain.

\section{QSO B0215+015}

The optical variability history from 1954 to 1982 of this target has been reported by Gaskell (1982). During this period, QSO B0215+015 has varied at least by three and possibly four magnitudes. Light curves in the optical domain during the periods 1981-1985 and 1989-1993 can be found in Webb et al. (1988) and in Schramm et al. (1994). Intranight variability at optical and near-IR wavelengths has been reported by Brindle (1996). No information was found in SIMBAD about the morphology of the target.

\section{QSO B0235+164}

This BL Lac object is a very well known and interesting object from an astrophysical point of view. UBVRI light curves are given by Raiteri (Raiteri et al. 2001, 2006) during the periods 1975 to 2000 and 2004 to 2005. The environment of QSO B0235+164 is a very complex one in the optical domain at the distance of a few arcseconds (Nilsson et al. 1996; O'Dowd \& Urry 2005). Bright structures extend 3 arcsec from the nucleus $(30 \mathrm{Kpc})$. The host galaxy analysis from the HST images (O'Dowd \& Urry 2005) leads to the conclusion that it is an unresolved host galaxy.

\section{QSO B0300+470}

Abraham et al. (1991) report that this BL Lac object is unresolved with the William Herschel Telescope (4.2 m located in La Palma) in the $R$ band. No information was found about magnitude variation in the optical domain.

\section{QSO B0309+411}

No information was found about the morphology and magnitude variation of this Seyfert I object in the optical domain.

\section{QSO B0332-403}

No information was found about the morphology and magnitude variation of this BL Lac object in the optical domain. 


\section{QSO B0403-132}

The host galaxy of this QSO has been detected by Ronnback et al. (1996) and Orndahl \& Ronnback (2005) in the optical band. No information was found about the magnitude variation in the optical domain.

\section{QSO B0405-123}

This Seyfert I galaxy is optically variable. It has been observed to vary by up to one magnitude over a seven-year period and to undergo repeated outbursts of about 0.3 mag over a period of 75-85 days (Bozyan et al. 1990). The host galaxy has been resolved in the $R$ band, and the closest apparent companion lies at a distance of 2.18 arcsec (Boyce et al. 1999).

\section{QSO B0422+004}

This BL Lac object was found to be resolved by Abraham et al. (1991) and Falomo (1996) and was unresolved by Wright et al. (1998) and Nilsson (2003). No information was found about the magnitude variation in the optical domain.

\section{QSO B0454+844}

This BL Lac object remains unresolved for Pursimo et al. (2002), Scarpa et al. (2000a), Urry et al. (2000), and Scarpa et al. (2000b). No information was found about the magnitude variation in the optical domain.

\section{QSO B0454-463}

This QSO is unresolved in the near-IR $H$-band by Kotilainen et al. (1998a). No other information was found about morphology and magnitude variation in the optical domain.

\section{QSO B0506-612}

No information was found about the morphology and magnitude variation of this QSO in the optical domain.

\section{QSO B0528-250}

This QSO has a very close companion at the angular distance of 1.17 arcsec on HST images (Moller \& Warren 1998). It is a variable object. Observations shows that it has decreased in brightness by two magnitudes in seven months (Jauncey et al. 1978). It also changes in brightness by a few tenths of a magnitude on timescales of one to a few days (Adam 1985). No information has been found about its morphology in the optical domain.

\section{QSO B0606-157}

This QSO is not a well-defined object in the optical domain. It seems that a host galaxy exists (Hutchings et al. 1988) with a faint halo in the $R$ band. A galaxy can be seen 3 arcsec away from the target (Hutchings et al. 1984). No information was found about variability in the optical domain.

\section{QSO B0636+680}

There is no information about the morphology and magnitude in the optical domain for this QSO.

\section{QSO B0716+714}

This BL Lac object is a very well known object in the optical-IR domain. The host galaxy is not resolved with the HST images in the optical domain (Urry et al. 2000) but is resolved in the $I$ band (Nilsson et al. 2008) with the NOT telescope in La Palma ( $I$ band magnitude of 17.5 and effective radius of 2.7 arcsec). This target is also very well known for its variability on different timescales, from intraday (Carini et al. 2011) to 50 years (Nesci et al. 2005).
QSO B0754+100

This BL Lac object is not resolved in the HST images (Scarpa et al. 2000a) in $V$ and $R$ bands. Nevertheless, it was resolved by Falomo (1996) in the $R$ band with the NTT telescope of ESO and by Abraham et al. (1991) also in the $R$ band with the William Herschel Telescope in La Palma. It is resolved by Heidt et al. (2004) in the I band with high-resolution images of the NOT telescope in La Palma. No information about the magnitude variation in the optical domain.

\section{QSO B0808+019}

This BL Lac object is not seen to be resolved by Abraham et al. (1991) in the $R$ band with the William Herschel Telescope in La Palma. No information was found for the magnitude variation in the optical domain.

\section{QSO B0823-223}

This BL Lac object is not seen to be resolved by Falomo (1996) in the $R$ band with the NTT of ESO. With a redshift of $z=0.910$, the host galaxy is expected to be completely hidden by the light of the BL Lac nucleus, even with subarcsec resolution. This target is a candidate for gravitational lensing. There is a galactic star located 1.5 arcsec west of the BL Lac. No information was found about the magnitude variation in the optical domain.

\section{QSO B0827+243}

Fan et al. (2004) observed this QSO with a $70 \mathrm{~cm}$ telescope in southwestern Georgia, and they give an overview of the available photometric data (not a lot before 1997). The target is variable in $V$ and $R$ both on short (Dai et al. 2001) and long timescales. No information was found about the morphology of the target in the optical domain.

\section{QSO B0834+250}

This QSO is not a very well known object. SIMBAD refers to 34 papers between 1850 and 2011 that have no information about the variabilty or morphology of this target in the optical domain.

\section{QSO B0912+297}

Zekl et al. (1981) gives a light curve of this BL Lac from 1910 to 1960 , as does Fiorucci \& Tosti (1996) between 1994 and 1995 ( $R$ band). Other references between 1972 and 1975 were given by Bozyan et al. (1990). No information was found about the morphology of the target in the optical domain.

\section{81}

Although this target is an ICRF source chosen for the link of the reference systems at radio wavelengths, it is obviously not a QSO in the optical domain.

\section{QSO B0954+658}

The host galaxy of this BL Lac is marginally resolved (the source is definitely resolved, but the data are insufficient for determining a firm Hubble class) on the Gunn $r$ band images of the CFHT (Wurtz et al. 1996). QSO B0954+658 is a well studied BL Lac object in the optical band. Raiteri et al. (1999) gives light curves in the $B$ and $V$ bands on long timescales (several years) together with radio curves.

\section{QSO B0955+326}

Adaptive optics imaging of this Seyfert I galaxy by Marquez et al. (2001) in the near-IR $H$ and $K$ bands shows an extension to the southwest up to 5 arcsec from the target. The host galaxy in 
near-IR bands is an elliptical one. Sagar et al. (2004) and Stalin et al. (2004) study the intranight variability of QSO B0955+326.

QSO B1012+232

No information was found about morphology and magnitude variation in the optical domain.

\section{QSO B1022+194}

Gehren et al. (1984), with the MPIA $2.2 \mathrm{~m}$ telescope at the Calar Alto observatory, gives provisional conclusions about the host galaxy in the Gunn $r$ band filter. The mean angular diameter is lower than 6 arcsec but no apparent magnitude is given. The underlying nebulosity seems to be heavily distorted. No information was found about magnitude variation in the optical domain.

\section{QSO B1039+811}

No information was found about morphology and magnitude variation in the optical domain.

QSO B1101+384

This BL Lac object is the Markarian galaxy Mrk 421. It is not a QSO in the optical domain.

QSO B1101-325

Some information exists in the optical domain for this Seyfert I galaxy, but conclusions about its variability and its morphology cannot be firmly drawn.

QSO B1111+149

This QSO is not a very well known object. SIMBAD refers to 55 papers between 1850 and 2011 without any information about the variability or morphology of this target in the optical domain.

QSO B1123+264

This QSO is not a very well known object. SIMBAD refers to 65 papers between 1850 and 2011 without any information about the morphology of this target in the optical domain. Miller (1983) gives a light curve in the blue band from 1928 to 1952.

\section{QSO B1144+402}

There is only one reference (Vigotti et al. 1989) for this QSO about morphology. The proposed optical identification is "galaxy". No information was found for the magnitude variation in the optical domain.

\section{QSO B1147+245}

This BL Lac object is not resolved by the HST (Urry et al. 2000) in the $R$ band. Neither Pursino et al. (2002) nor Stickel et al. (1993) have resolved this object. No information was found about magnitude variation in the optical domain.

QSO B1215+303

Gopal-Krishna et al. (2003) report intranight variability in the $R$ band with the $1.04 \mathrm{~m}$ Sampurnanand telescope (India), as does Stalin et al. (2004) for the long-term optical variability. Sbarufatti et al. (2005) refer to a host galaxy with known redshift.

\section{QSO B1219+044}

No information was found about morphology and magnitude variation in the optical domain.

\section{84}

This Seyfert II galaxy is known as M 84 and is obviously not a compact object in the optical domain.

\section{QSO B1252+119}

The light curve of this QSO during 21 years of observation (1969 to 1990) is given by Smith et al. (1993). They use the $76 \mathrm{~cm}$ reflector of the Rosemary Hill Observatory in the international photographic system. During this period the target shows a peak-to-peak variation of about one magnitude. No information was found for the morphology in the optical domain.

\section{ICRF J133245.2+472222}

Nineteen papers relating to this QSO are found in SIMBAD, but none of them refers to magnitude variation or morphology in the optical domain.

\section{ICRF J135256.5-441240}

Confusing data have been published about the optical identification of the BL Lac ICRF J135256.5-441240 and the Seyfert I galaxy QSO B1349-439 (Veron 1996). This BL Lac was observed by Heidt et al. (2004) with the ESO-VLT on Cerro Paranal, Chile. They could not resolve the target in the $I$ band. No information was found for the magnitude variation in the optical domain.

\section{QSO B1416+067}

This QSO is resolved by Lehnert et al. (1999) with the HST images in the $R$ band. No information was found about magnitude variation in the optical domain.

\section{QSO B1420+326}

No information was found about morphology and magnitude variation in the optical domain.

\section{QSO B1424+240}

Scarpa et al. (2000a) did not resolve this BL Lac in the HST images in the $R$ band, but Katajainen et al. (2000) provide a light curve in the $V$ band from 1995 to 1997.

\section{QSO B1508-055}

There is only one paper about the morphology of this QSO by Francis et al. (2000). The morphology (point) of this target was retrieved from the classification by the COSMOS platemeasuring machine of the UK Schmidt plates and checked by visual inspection of the images. These authors give also the magnitude of the target in $B, V, R, I, J, H$, and $K$ bands.

\section{QSO B1519-273}

High-resolution images of this BL Lac in the $I$ band with the VLT show that it is unresolved (Heidt et al. 2004). Nevertheless, the analysis was seriously hampered by the presence of a star 0.8 arcsec from the target. Scarpa et al. (2000a) observed it with the HST in the $R$ and $V$ bands and came to the same conclusion about the unresolvability. Fan (1997) gives a $V$ band magnitude variation of 2.43 .

\section{QSO B1538+149}

The host galaxy of this BL Lac is resolved in optical bands by Scarpa et al. (2000a), but it is morphologically unclassified. The host galaxy remains unresolved in the near-IR (Kotilainen et al. 1998b). From 1979 to 1986 the target shows a 1.28 mag variation in the $B$ band (Pica et al. 1988). Xie et al. (1990) report an intranight variability of about 0.5 mag in the $V$ band. 
QSO B1546+027

From 1971 to 1983 this QSO varies by about 0.93 photographic magnitudes (Pica et al. 1988). Malkan (1984) resolves the host galaxy with the Palomar 1.5 m telescope (Gunn $v, g, r$ filters).

QSO B1637+574

Orndahl et al. (2003) in the $R$ band with the NOT telescope in La Palma does not resolve the host galaxy. No information was found about magnitude variation in the optical domain.

\section{QSO B $1725+044$}

There is only one paper about the morphology of this Seyfert I galaxy by Francis et al. (2000). The morphology (point) of this target was retrieved from the classification by the COSMOS plate-measuring machine of the UK Schmidt plates and checked by visual inspection of the images. Francis also gives the magnitude of the target in $B, V, R, I, J, H$, and $K$ bands.

\section{QSO B $1727+502$}

Katajainen et al. (2000) report an optical magnitude variation for this BL Lac in previous studies. Its $V$ band observations show a 0.36 magnitude variation. Optical $R$ band images obtained at the Nordic Optical Telescope by Nilsson et al. (2003) show that the host galaxy is resolved in the same way as for the images obtained with the CFHT by Wurtz et al. (1996) in Gunn $r$ filter.

\section{QSO B1749+096}

This BL Lac in a crowded field is resolved, but morphologically unclassified, with the HST in Cousins $R$ filter by Scarpa et al. (2000a). Several stars surround this low galactic latitude object, which makes it difficult to study from the ground. Xie et al. (2002) report intranight variability of about $0.69 \mathrm{mag}$ in $43 \mathrm{~min}$.

\section{QSO B1800+440}

No information was found about morphology and magnitude variation in the optical domain.

\section{QSO B1849+670}

There were only 61 papers about this target between 1850 and 2011. No information was found about morphology and the magnitude variation in the optical domain.

\section{ICRFJ192332.1-210433}

There were only 45 papers about this target between 1850 and 2011. No information was found about morphology and magnitude variation in the optical domain. Costa (2001) proposes a new optical identification of this QSO.

\section{QSO B1954-388}

The host galaxy of this QSO has been resolved by Kotilainen et al. (1998a) in the near-IR $H$ band with the ESO $2.2 \mathrm{~m}$ telescope of the ESO in La Silla. The point-like morphology of this target in the optical bands (Francis et al. 2000) was retrieved from the classification by the COSMOS plate-measuring machine of the UK Schmidt plates and checked by visual inspection of the images. Francis also gives the magnitude of the target in $B, V, R, I, J, H$, and $K$ bands.

\section{QSO B2059+034}

From 1971 to 1991 this QSO varies by about 1.2 photographic magnitudes (Smith et al. 1993). The point-like morphology of this target in the optical bands (Francis et al. 2000) was retrieved from the classification by the COSMOS plate-measuring machine of the UK Schmidt plates and checked by visual inspection of the images. Francis also gives the magnitude of the target in $B, V, R, I, J, H$, and $K$ bands.

\section{QSO B2126-158}

The point-like morphology of this QSO in the optical bands (Francis et al. 2000) was retrieved from the classification by the COSMOS plate-measuring machine of the UK Schmidt plates and checked by visual inspection of the images. Francis gives also the magnitude of the target in $B, V, R, I, J, H$, and $K$ bands. Borgeest \& Schramm (1994) did not see any variability in the $R$ band for this QSO. No other reference was found among the 348 papers between 1850 and 2011.

\section{QSO B2143-156}

Netzer et al. (1996) gives a light curve in $R$ and $B$ bands obtained during 3.5 years at the Wise Observatory ( $1 \mathrm{~m}$ telescope in Mitzpe Ramon, Israel), CTIO, and McDonald Observatory (36 or 30 inch telescopes). The curves shows a variation of $0.39 \mathrm{mag}$ in the $B$ band and $0.24 \mathrm{mag}$ in the $R$ band, both with a constant slope. The point-like morphology of this Seyfert I galaxy in the optical bands (Francis et al. 2000) was retrieved from the classification by the COSMOS plate-measuring machine of the UK Schmidt plates and checked by visual inspection of the images. Francis also gives the magnitude of the target in $B, V, R, I, J, H$, and $K$ bands.

\section{QSO B2155-304}

This BL Lac is a target of interest from the astrophysical point of view (1031 papers between 1850 and 2011). Dominici et al. (2004) give a light curve in the $V$ band for the period 1996-1999 where variations of one magnitude in a few days can be seen. These authors also produced a lot of reference papers about the variability of this target. Dolcini et al. (2007) observe the target in near IR and give light curves in the $J, H$, and $K$ bands between May and December 2005. Fan \& Lin (2000b) give historical light curves in $U, B, V, R$, and $I$ bands from 1970 to 2000 and find a possible periodicity of 4.16 years and 7.0 years. Kotilainen et al. (1998b) resolve the host galaxy in the near-IR $H$ band with the $2.2 \mathrm{~m}$ telescope at ESO. Nevertheless for Wurtz et al. (1996) the host galaxy remains unresolved in the $R$ band with the CFH Telescope.

\section{QSO B2227-088}

Falomo et al. (2004) used the ESO VLT UT1 8m telescope under excellent seeing conditions to resolve the host galaxy in the $K$ band. The observed radial brightness profile of this QSO is nevertheless very near to the one of the PSF. The point-like morphology of this QSO in the optical bands (Francis et al. 2000) was retrieved from the classification by the COSMOS plate-measuring machine of the UK Schmidt plates and checked by visual inspection of the images. These authors also give the magnitude of the target in $B, V, R, I, J, H$, and $K$ bands.

\section{QSO B2250+194}

They were only 22 papers about this target between 1850 and 2011. There is no information about morphology and the magnitude variation in the optical domain.

\section{QSO B2254+024}

No information was found about morphology and magnitude variation in the optical domain. 
QSO B2254+074

Falomo et al. (1997) present high-resolution images of this BL Lac object with the WFPC2 camera of the Hubble Space Telescope. The host galaxy is a moderately bright elliptical one. The host galaxy is also resolved by Pursino et al. (2002) in the $R$ band. Fan \& Lin (2000a) report magnitude variations in the $U$, $B, V, R$, and $I$ bands after studying historical light curves. The greater variation (3.09) occurred in the $B$ band and the lower (0.93) in the $I$ band.

\section{QSO B2255-282}

No information was found about morphology and magnitude variation in the optical domain.

\section{QSO B2300-683}

Garcia et al. (1999) give a light curve in the $V$ band obtained with the $0.60 \mathrm{~m}$ Telescope located at the Observatorio do Pico dos Dias (Brazil). The time span is from March 1993 to July 1996. The target shows an amplitude variation of about 1.4 mag. No information was found about morphology in the optical domain.

\subsection{Light curves}

Objects in the northern hemisphere were observed with the T120 and TAROT(OCA) telescopes, whereas the targets of the southern hemisphere were observed with the Zadko and the TAROT(ESO) telescopes. All these instruments were presented Sect. 2. The kick off of the observations for the OHP and TAROT telescopes were respectively in March 2010 and February 2011. The Zadko telescope began observing in June 2011. Because of technical problems with the dome of the observatory, the number of observations obtained with this last instrument are not at the same level as for the two other robotic telescopes. The OHP telescope is manipulated by hand so that the number of observations is obviously always lower than the number of observations obtained with a robotic telescope. Figures 1 to 4 give some examples of light curves obtained with the TAROT telescopes (average magnitude was subtracted for clarity). All observations presented in the frame of this work were made before 6 January 2012. They are presented in Table 4 and will be part of the data provided to users through a database. This database is currently under construction in the framework of the ICRS-PC and will be the subject of a forthcoming paper.

It is a fact that the variation in the AGN's radiostructure degrades the positional accuracy of the radiocenter's position. Instabilities/variability in AGN components may degrade the photocenter accuracy. For example, it is well known that changes in the radio structure, such as those detected in VLBI, may degrade the radiocenters position. Also in the optical domain, instabilities in the accretion disk may contribute to a change in the optical photocenter (Popovic et al. 2012). We note that the photocenter changes due to variability in regions as small as $1 \mathrm{pc}$ (that include accretion disk and broad emission line regions) in objects towards $z \sim 0.3$ (corresponding to angular sizes over $200 \mu$ as) may, putatively, be detected with Gaia.

Figure 1 gives an example of a light curve for a wellobserved target (Katajainen et al. 2000), QSO B0716+714. Our light curve shows a $1.5 \mathrm{mag}$ variation (in both $R$ and $V$ bands) during one month. Due to the distance of this target $(z=0.3)$, it means that the emission region has an angular (maximum) size of roughly $5 \mu$ as. Other curves (Katajainen et al. 2000) show the same kind of events but during three month (15 $\mu$ as). Other targets from our work show magnitude variations during three months (for example $1147+245, z=0.2,25 \mu \mathrm{as}$ ).
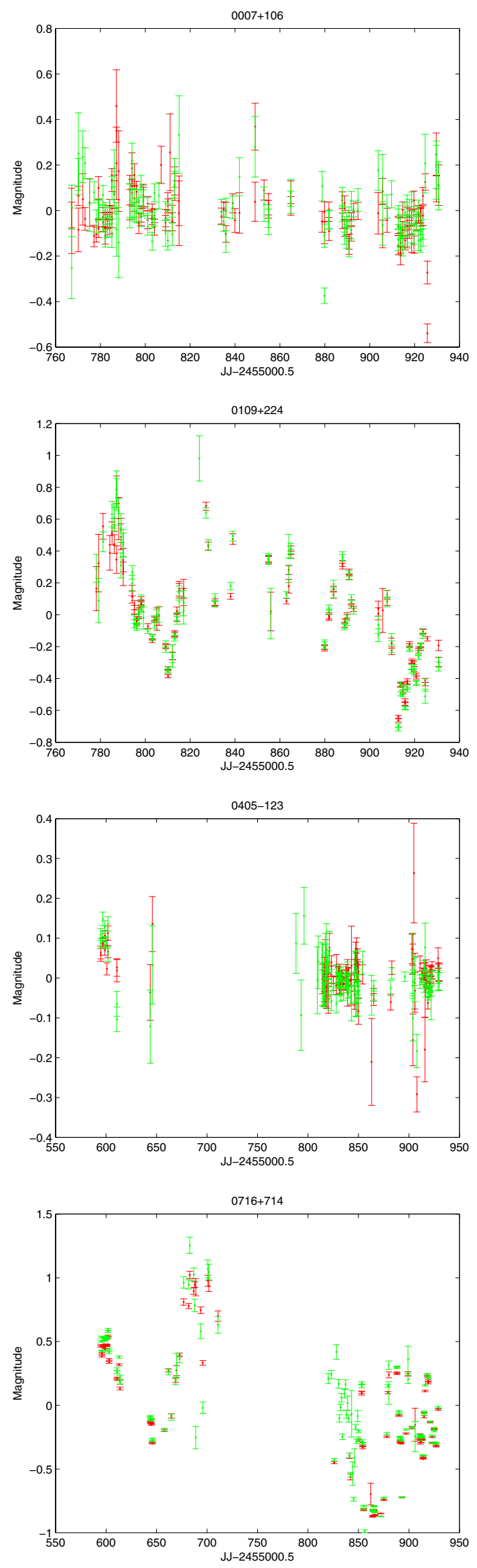

Fig. 1. Comparison between $G$ mag (green) and $R$ mag (red) for QSO B0007+106, QSO B0109+224, QSO B0405-123, and QSO B0716+714. 
F. Taris et al.: Optical monitoring of extragalactic sources
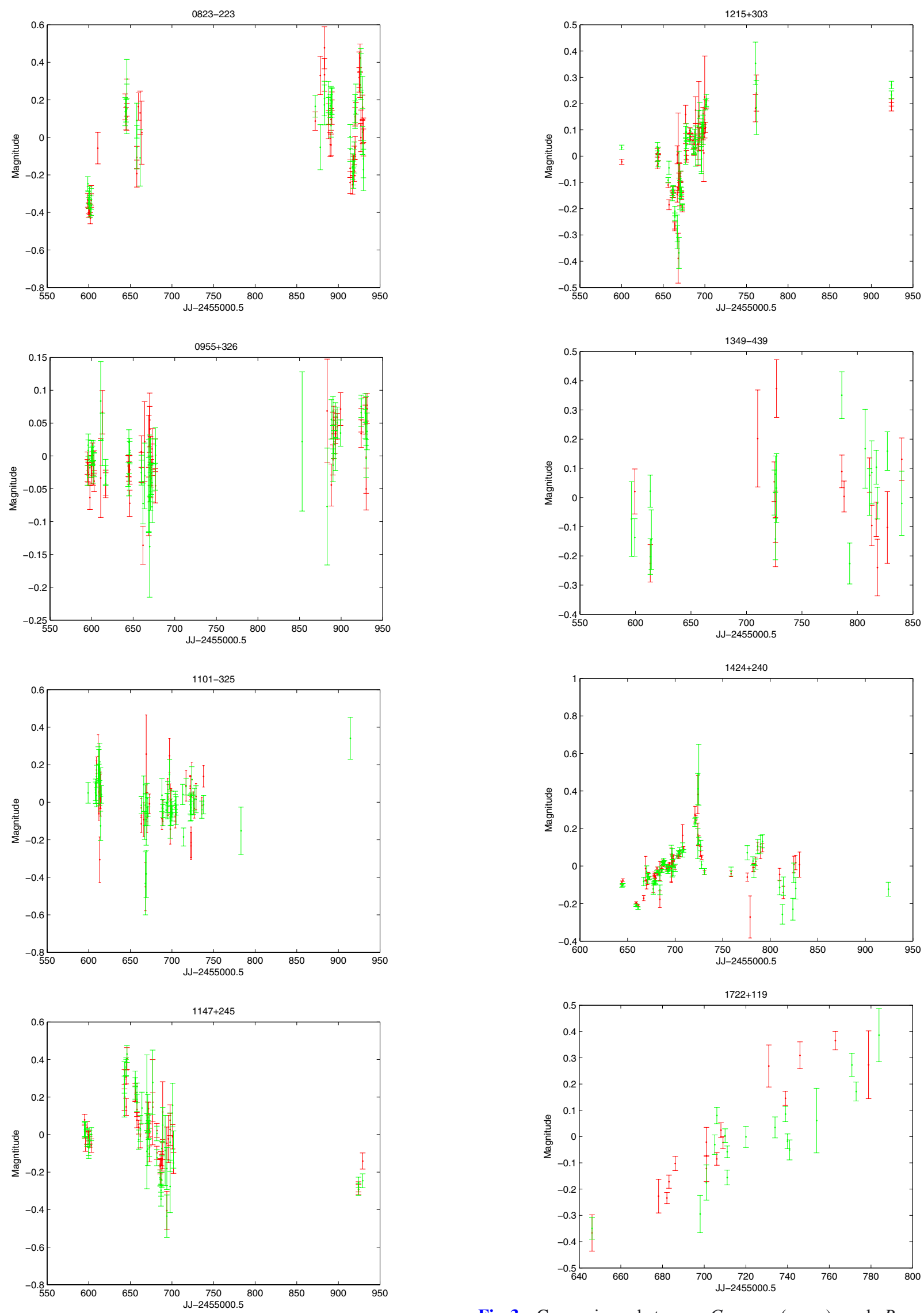

Fig. 2. Comparison between $G$ mag (green) and $R$ mag (red) for QSO B0823-223, QSO B0955+326, QSO B1101-325, and QSO B1147+245. 


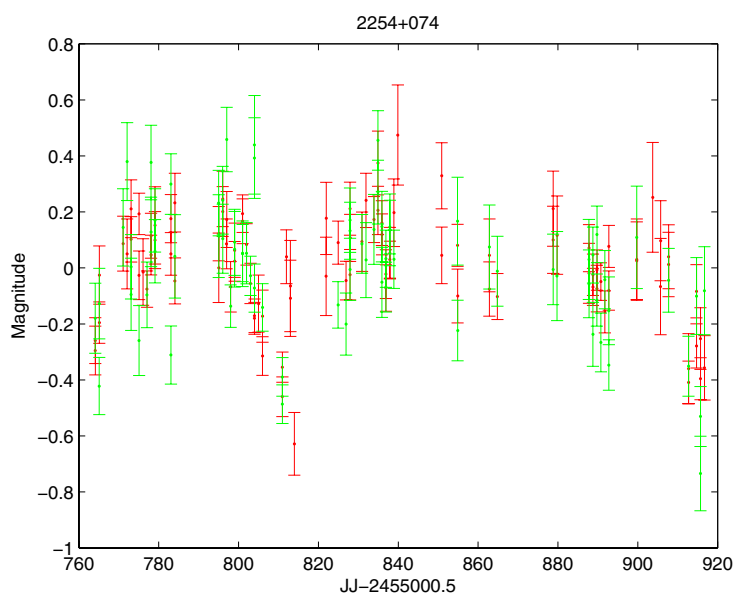

Fig. 4. Comparison between $G$ mag (green) and $R$ mag (red) for QSO 2254+074.

\section{Morphological analysis}

The pixelization from the OHP, Zadko, and both Tarot telescopes does not enable a detailed morphological investigation of the targets. However, early analysis using the second-generation Digitized Sky Survey images ${ }^{11}$ of several thousand quasars has shown that cases of extensive apparitions of the host galaxy can be spotted progressively from the bluer to the redder images (Souchay et al. 2012). Thus the same analysis is applied here, with the added advantage of repetating observations for the sake of obtaining the light curve and the repeatability of the CCD response.

The essence of the method is that the presence of the host galaxy can also be inferred by departures from the compound point spread function (PSF) of the quasar and host galaxy to the purely pointlike stellar PSFs (Falomo et al. 2001). We thus derive a PSF-based morphological classification of the objects using the available images in different filters. The morphological classification is obtained from comparing the quasar PSF to the local PSF. To that, neighborhoods of $5 \times 5$ arcmin around the quasar are obtained independently of the each image. The IRAF task DAOFIND is used to find both stars and the target, as well as to derive the PSF parameters. Stars are collected within one magnitude of the quasars magnitude, but in case fewer than five stars are picked up, the magnitude limits are progressively enlarged in one-magnitude steps, except for stars brighter than the tenth magnitude. Stars must be isolated from each other by an inner radius of ten pixels and within the frame by the same threshold. If fewer than five comparison stars are found no morphological index is derived for that quasar on that frame. Three estimators of the PSF are used: SHARP (probing skewness), SROUND (probing roundness), and GROUND (probing normalness). The morphological indexes are given by

$I_{\mathrm{PC}}(\mathrm{Q})=\left|\frac{P_{\mathrm{Q}}-\overline{P_{\mathrm{S}}}}{\sigma_{\mathrm{S}}}\right|$

where $I$ is the morphological index of quasar Q for the PSF parameter $\mathrm{P}$ in the color $\mathrm{C}$, given in comparison to the mean value from the stars $\mathrm{s}$, and normalized by the stellar standard deviation $\sigma$.

\footnotetext{
${ }^{11}$ http://archive.stsci.edu/dss/data.html
}

Table 1. Filters and pixel scale for the telescopes used in this program.

\begin{tabular}{lccc}
\hline \hline Telescope & OHP & Zadko & Tarots OCA/ESO \\
Filters & $V, R$ & $C, g, r$ & $C, V, R$ \\
Pixel Scale (") & 0.684 & 0.673 & 3.240 \\
\hline
\end{tabular}

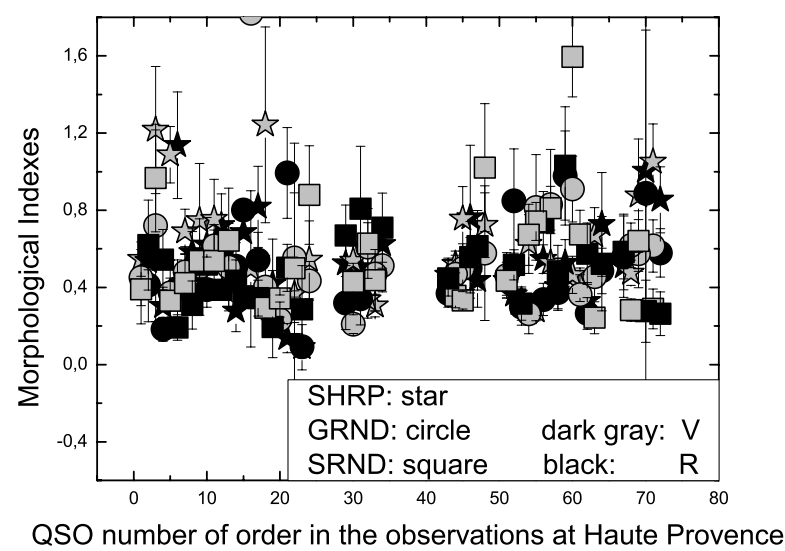

Fig. 5. Morphological indexes for the Haute Provence telescope. The morphological indexes are SHRP (stars, probing skewness), GRND (circles, probing normalness), and SRND (squares, probing roundness). The filters are indicated by different shades, light gray for clear, gray for visible or green, and black for red. The order number was assigned by increasing right ascension, and empty patches reflect sources with few observations for which the average was not attempted.

The telescope filters and pixelization are shown in Table 1. As it can be noticed, there is a lack of blue and near-IR colors that limits the analysis of the progressive separation on color of the quasar's PSF from a stellar one. On the other hand, the pixelization is not much worse than the DSS one, except for the Tarot telescopes.

The OHP's geographical location, as well as its pixel scale, enabled the best possible evaluation of the morphological results. The quasars observed at the OHP can be divided into (i) 16 objects appearing in the SDSS and there classified both as quasars and point sources and (ii) 49 objects that do not belong in the SDSS as quasars (some might appear under other classifications). All targets common to the SDSS are found here as not distinguished from the PSF given by local stars. Among the nonSDSS objects, there are 11 Seyfert1, 23 BL Lac, and 15 QSO objects as classified from the CDS database. From there the images can be examined (mostly taken from the DSS) and for none of them the host galaxy has been seen. Nonetheless, among those objects (like for example B0007+106 or B0954+658, respectively classified as Seyfert1 and BL Lac), host galaxy had been previously resolved in more favourable conditions of SNR and pixelization. That there is a resolvable host galaxy that goes undetected in our sample indicates that, although those and other such objects will probably remain in the GCRF, they will probably not be in the core Gaia Fundamental Quasar Reference Frame. This underlines the need for previous and in-mission variability and morphological surveying.

The plots in Figs. 5 to 8 give the three morphological indexes for every source presented in Table 4, as an unweighted average of the values from the various measures taken to build the light curves. The morphological indexes, in each color and for each source, are displayed along the same vertical axis, following the order given in the abscissa. These sources have been observed by 


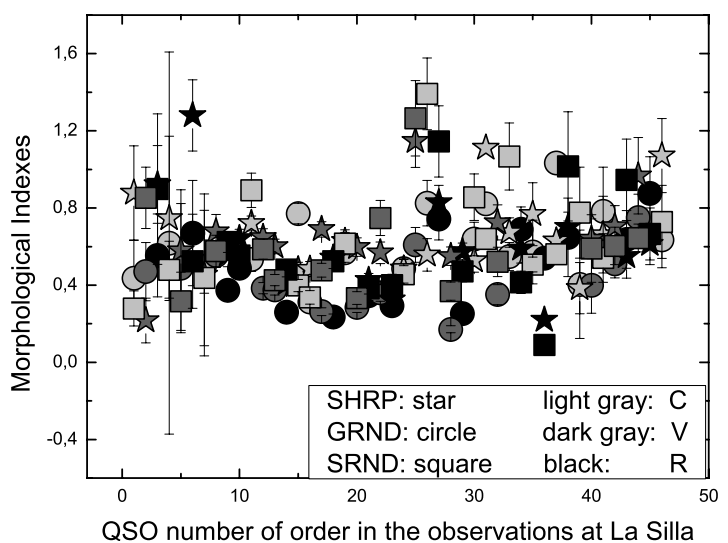

Fig. 6. Morphological indexes for the Tarot Chile telescope. The morphological indexes are SHRP (stars, probing skewness), GRND (circles, probing normalness), and SRND (squares, probing roundness). The filters are indicated by different shades, light gray for clear, gray for visible or green, and black for red. The order number was assigned by increasing right ascension, and empty patches reflect sources with few observations for which the average was not attempted.

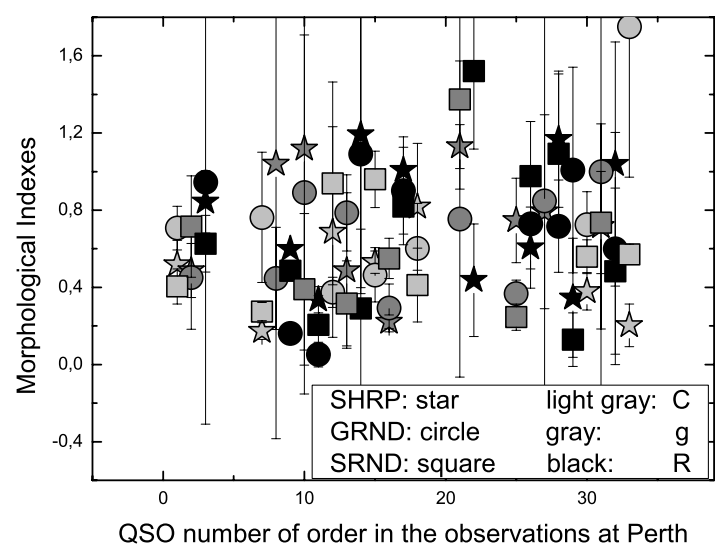

Fig. 7. Morphological indexes for the Zadko telescope. The morphological indexes are SHRP (stars, probing skewness), GRND (circles, probing normalness), and SRND (squares, probing roundness). The filters are indicated by different shades, light gray for clear, gray for visible or green, and black for red. The order number was assigned by increasing right ascension, and empty patches reflect sources with few observations for which the average was not attempted.

the four telescopes as shown in Table 3 . The three morphological indexes are displayed by different symbols, while different shades distinguish the filters used. The errors bars are the standard error of the mean. They are clearly small, which shows the consistency of the morphological indexes from observation to observation. As discussed above, the available pixelization does not enable one to clearly signal the fuzzy host galaxy of individual quasars, but their ensemble does support the validity of the method. On one hand it is noticeable that on the very large pixel scale of the Tarots telescopes, all targets at all morphological indexes are tightly packed close to zero, which means that they are pointlike. For the Haute-Provence and Zadko telescopes, the range is clearly wider, indicating that a fraction of the objects should present the isophotes of the host galaxy in conditions of a finer pixel scale and sharper PSF. Also the filters' range is not broad enough to really separate the bluer center from the redder stellar-contaminated component. Nevertheless, the finest pixelization at the Zadko telescope means that larger

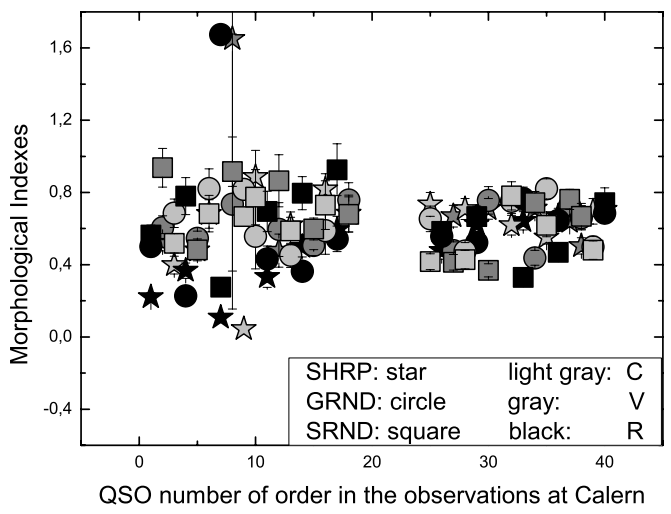

Fig. 8. Morphological indexes for the Tarot Calern telescope. The morphological indexes are SHRP (stars, probing skewness), GRND (circles, probing normalness), and SRND (squares, probing roundness). The filters are indicated by different shades, light gray for clear, gray for visible or green, and black for red. The order number was assigned by increasing right ascension, and empty patches reflect sources with few observations for which the average was not attempted.

morphological indexes appear for the $R$ filter than for the $g$ filter. The best determination of the morphological indexes for these sources, calculated by the method outlined above, is found in Souchay et al. (2012).

\section{Conclusion and prospects}

In the first part of this paper we presented the telescopes used for this experiment, particularly robotic telescopes of little or medium aperture $(0.25 \mathrm{~m}$ to $1 \mathrm{~m})$. This kind of telescope is well suited to regular monitoring of the quasar's flux. The sampling of the observations (1 day) allow reaching physical events of small size (1 light-day, region near the black hole), and the longer periods, possibly representing physical events of larger dimensions, are reachable thanks to the total span of observation (the maximum size of an object varying in some characteristic time is equal to the distance covered by the light during that time). Considering the redshift of our target set, the corresponding astrometric variations would be near some $\mu$ as to the level of the mas. This level of uncertainty must be compared to the astrometric uncertainty expected for the final Gaia catalog. All the targets observed during this experiment have relatively bright magnitude (brighter than 18) so are observable by our small telescopes. Our experiment then clearly shows the utility of such telescopes for the magnitude monitoring of AGNs.

In the second part of this paper, notes about individual targets were presented and summarized in terms of variability and morphology. Light curves obtained with the four telescopes involved in these observations were also presented. For 36 targets (54\% of the initial list), the light curves presented in this work bring new information. Our curves clearly show magnitude variations with a strong correlation between the different filters used. Some targets are also observed by other groups and show the same kind of event in terms of the time duration and amplitude of the variation. For QSO B0716+714 Katajainen et al. (2000) also report an event with a period of 60 to 70 days. For the well-sampled curves, the white phase noise is never present as we might expect for measurement noise. On the contrary, there are always characteristic trends in physical processes on all time scales (day to month/year) indicating that the morphology of the target could be influenced by intrinsic or extrinsic physical processes with 
Table 2. Summary of information on host galaxies and the variability found for optical bands in the SIMBAD database.

\begin{tabular}{|c|c|c|c|}
\hline Name & Type & Host galaxy & Variability \\
\hline QSO B0007+106 & Seyfert I & yes & yes \\
\hline QSO B0010+405 & Seyfert I & yes & unknown \\
\hline QSO B0048-097 & BL Lac & no & yes \\
\hline QSO B0109+224 & BL Lac & no & yes \\
\hline $\mathrm{QSO} \mathrm{B} 0202+319$ & QSO & unknown & unknown \\
\hline QSO B0208-512 & BL Lac & unknown & unknown \\
\hline QSO B0215+015 & QSO & unknown & yes \\
\hline QSO B0235+164 & BL Lac & no & yes \\
\hline QSO B0300+470 & BL Lac & no & unknown \\
\hline QSO B0309+411 & Seyfert I & unknown & unknown \\
\hline QSO B0332-403 & BL Lac & unknown & unknown \\
\hline QSO B0403-132 & QSO & yes & unknown \\
\hline QSO B0405-123 & Seyfert I & yes & yes \\
\hline QSO B0422+004 & BL Lac & yes* & unknown \\
\hline QSO B0454+844 & BL Lac & no & unknown \\
\hline QSO B0454-463 & QSO & unknown & unknown \\
\hline QSO B0506-612 & QSO & unknown & unknown \\
\hline QSO B0528-250 & QSO & unknown & yes \\
\hline QSO B0606-157 & QSO & yes* & unknown \\
\hline QSO B0636+680 & QSO & unknown & unknown \\
\hline QSO B0716+714 & BL Lac & no & yes \\
\hline QSO B0754+100 & BL Lac & yes* & unknown \\
\hline QSO B0808+019 & BL Lac & no & unknown \\
\hline QSO B0823-223 & BL Lac & no & unknown \\
\hline QSO B0827+243 & QSO & unknown & yes \\
\hline QSO B0834+250 & QSO & unknown & unknown \\
\hline QSO B0912+297 & BL Lac & unknown & yes \\
\hline QSO B0954+658 & BL Lac & yes & yes \\
\hline QSO B0955+326 & Seyfert I & unknown & yes* \\
\hline QSO B1012+232 & Seyfert I & unknown & unknown \\
\hline QSO B1022+194 & QSO & yes & unknown \\
\hline QSO B1039+811 & QSO & unknown & unknown \\
\hline QSO B1101-325 & Seyfert I & unknown & unknown \\
\hline QSO B1111+149 & QSO & unknown & unknown \\
\hline QSO B1123+264 & QSO & unknown & yes \\
\hline QSO B1144+402 & QSO & yes* & unknown \\
\hline QSO B1147+245 & BL Lac & no & unknown \\
\hline QSO B1215+303 & BL Lac & yes & yes \\
\hline QSO B1219+044 & QSO & unknown & unknown \\
\hline QSO B1252+119 & QSO & unknown & yes \\
\hline ICRF J133245.2+472222 & QSO & unknown & unknown \\
\hline ICRF J135256.5-441240 & BL Lac & unknown & unknown \\
\hline QSO B1416+067 & QSO & yes & unknown \\
\hline QSO B1420+326 & QSO & unknown & unknown \\
\hline QSO B1424+240 & BL Lac & no & yes \\
\hline QSO B1508-055 & QSO & yes* & yes* \\
\hline QSO B1519-273 & BL Lac & no & yes \\
\hline QSO B1538+149 & BL Lac & yes & yes \\
\hline QSO B1546+027 & QSO & yes & yes \\
\hline QSO B1637+574 & Seyfert I & no & unknown \\
\hline
\end{tabular}

Notes. In the column host galaxy yes indicates that the host galaxy is known and resolved in the optical band. A yes* refers to some comments in the text. In the same manner, a yes in the column variability means that the target has at least one measure of magnitude (regardless of the optical band). Unknown means that no information was found in the SIMBAD database whether for morphology or for variability.
Table 2. continued.

\begin{tabular}{lccc}
\hline \hline Name & Type & Host galaxy & Variability \\
\hline QSO B1725+044 & Seyfert I & yes* & yes* \\
QSO B1727+502 & BL Lac & yes & yes \\
QSO B1749+096 & BL Lac & yes & yes* \\
QSO B1800+440 & QSO & unknown & unknown \\
QSO B1849+670 & Seyfert I & unknown & unknown \\
ICRF J192332.1-210433 & QSO & unknown & unknown \\
QSO B1954-388 & QSO & yes* & yes* \\
QSO B2059+034 & QSO & yes* & yes \\
QSO B2126-158 & QSO & yes* & yes* \\
QSO B2143-156 & Seyfert I & yes* & yes \\
QSO B2155-304 & BL Lac & no & yes \\
QSO B2227-088 & QSO & yes* & yes* \\
QSO B2250+194 & Seyfert I & unknown & unknown \\
QSO 2254+024 & QSO & unknown & unknown \\
QSO B2254+074 & BL Lac & yes & yes \\
QSO B2255-282 & Seyfert I & unknown & unknown \\
QSO B2300-683 & Seyfert I & no & yes \\
\hline
\end{tabular}

an angular value close to the astrometric uncertainty of the final Gaia catalog.

Among the 70 initial targets, roughly $50 \%$ have an unknown or not well-established morphology. Seventeen targets have high-resolution images coming from the Hubble Space Telescope and are currently under investigation in the search for the host galaxy. Twenty-two other targets have no information and will be the subject of dedicated high-resolution observations to find their morphology.

In the third part of this paper we showed that the morphological indexes of the targets do not vary above the noise, as obtained from the images coming from these four telescopes and detectors. The results we obtained are in good agreement with other studies based on DSS or SDSS images. We showed that the quasars are a special group of objects compared to the stars used for comparing the PSF parameters.

The detailed results for these morphological indexes, their numerical values, the stability of the time series of the indexes, and the comparison with the morphological analysis currently in progress with the GALFIT and IRAF software will be presented as second part of the present work.

The results in this paper are being pursued to monitor the target's magnitude as given in the first list of Bourda. Another list of targets has been established to achieve the link between the radio and the optical reference frame (Bourda et al. 2010). We then plan to observe this second set of targets coming from the NVSS (NRAO VLA Sky Survey) catalog but with other robotic telescopes because all these targets are spread over the northern hemisphere. The same kind of result are expected in terms of variability and morphology and will be published as third and fourth parts of the present work.

Our group is also involved in other works to study the morphology of AGN in the area of a Gaia Workpackage (GWP-S335-13000) together with the relationship between the radio and optical observations. For this last topic, dedicated observations are planned in these two wavelength domains. Furthermore, a database dedicated to AGN optical images is currently under construction in the framework of the Virtual Observatory and will be presented in a forthcoming paper. 
Table 3. Journal of observations.

\begin{tabular}{|c|c|c|c|c|c|}
\hline Name & ESO & OCA & OHP & Zadko & HST \\
\hline QSO B0007+106 & 34 & 183 & 8 & 15 & \\
\hline QSO B0010+405 & & & 8 & & \\
\hline QSO B0048-097 & & & 8 & & 9 \\
\hline QSO B0109+224 & 19 & 136 & 12 & 7 & \\
\hline QSO B0202+319 & & & 12 & & 4 \\
\hline QSO B0208-512 & & & & & 11 \\
\hline QSO B0215+015 & & 10 & 4 & & 1 \\
\hline QSO B0235+164 & & 8 & 20 & & 43 \\
\hline QSO B0300+470 & & & 10 & & \\
\hline QSO B0309+411 & & & 4 & & \\
\hline \multicolumn{6}{|l|}{ QSO B0332-403 } \\
\hline \multicolumn{6}{|l|}{ QSO B0403-132 } \\
\hline QSO B0405-123 & 82 & 55 & & & 22 \\
\hline QSO B0422+004 & & & 9 & & \\
\hline QSO B0454+844 & & & 4 & & 4 \\
\hline \multicolumn{6}{|l|}{ QSO B0454-463 } \\
\hline QSO B0506-612 & & & & & 4 \\
\hline QSO B0528-250 & & & & & 50 \\
\hline \multicolumn{6}{|l|}{ QSO B0606-157 } \\
\hline QSO B0636+680 & & & 4 & & 1 \\
\hline QSO B0716+714 & & 122 & & & 13 \\
\hline QSO B0754+100 & & 14 & & & 4 \\
\hline QSO B0808+019 & & & 9 & & \\
\hline QSO B0823-223 & 215 & 70 & & & \\
\hline QSO B0827+243 & & & 4 & & 10 \\
\hline QSO B0834+250 & & & 8 & & \\
\hline QSO B0912+297 & & 6 & 16 & & \\
\hline QSO B0954+658 & & & 14 & & 4 \\
\hline QSO B0955+326 & 280 & 129 & & & 36 \\
\hline QSO B1012+232 & & 18 & & & \\
\hline QSO B1022+194 & & 11 & & & \\
\hline QSO B1039+811 & & & 14 & & 4 \\
\hline QSO B1101-325 & 298 & & & 4 & \\
\hline QSO B1111+149 & & 5 & & & \\
\hline QSO B1123+264 & & & 21 & & \\
\hline QSO B1144+402 & & & 22 & & \\
\hline QSO B1147+245 & 223 & 197 & & 6 & 5 \\
\hline QSO B1215+303 & 119 & 190 & & 27 & 6 \\
\hline QSO B1219+044 & 7 & & & & \\
\hline QSO B1252+119 & & 6 & & & \\
\hline ICRF J133245.2+472222 & & & 21 & & \\
\hline ICRF J135256.5-441240 & 93 & & & 17 & \\
\hline QSO B1416+067 & & 129 & & 8 & 11 \\
\hline QSO B1420+326 & & & 20 & & \\
\hline QSO B1424+240 & 132 & 199 & 16 & 8 & 5 \\
\hline QSO B1508-055 & & & 14 & 16 & 1 \\
\hline QSO B1519-273 & 7 & & & & 4 \\
\hline QSO B1538+149 & & & 16 & & 29 \\
\hline QSO B1546+027 & & & 12 & 12 & \\
\hline QSO B1637+574 & & & 18 & & \\
\hline QSO B1725+044 & & & 16 & & \\
\hline QSO B1727+502 & & & 15 & & 4 \\
\hline
\end{tabular}

Notes. The number of observation in September 2011 is given for each telescope (ESO or OCA for the Tarots, OHP for the T120, and Zadko for the Zadko telescope) used in this work plus images obtained from the HST archive to search for the host galaxy.
Table 3. continued.

\begin{tabular}{lccccc}
\hline \hline Name & ESO & OCA & OHP & Zadko & HST \\
\hline QSO B1749+096 & 81 & 4 & 17 & 4 & 4 \\
QSO B1800+440 & & & 22 & & \\
QSO B1849+670 & & & 16 & & \\
ICRF J192332.1-210433 & 3 & & & & \\
QSO B1954-388 & 7 & & & 10 & \\
QSO B2059+034 & & & 12 & & \\
QSO B2126-158 & 211 & & & 13 & 1 \\
QSO B2143-156 & 240 & & & 6 & \\
QSO B2155-304 & 25 & & & 3 & 2 \\
QSO B2227-088 & & & 4 & & \\
QSO B2250+194 & & & 16 & & \\
QSO 2254+024 & & & 9 & & 1 \\
QSO B2254+074 & 56 & 199 & 8 & & 32 \\
QSO B2255-282 & 8 & & & 3 & 1 \\
QSO B2300-683 & 51 & & & & \\
\hline
\end{tabular}

Acknowledgements. We are very grateful to an anonymous referee for very useful comments and to R. Giraud from the "Observatoire de Haute Provence" for his help in the observation of our targets with the T120 telescope. The authors are also grateful to J. Adams for careful language editing of the manuscript. The French AS-Gaia is kindly acknowledged for the financial support of the observation campaign at the Observatoire de Haute Provence. A.H.A. acknowledges CNPq grant PQ306775/2009-3 and PARSEC International Incoming Fellowship of the Marie Curie 7th ECP.

\section{References}

Abraham, R., McHardy, M., \& Crawford, C. 1991, MNRAS, 252, 482 Adam, G. 1985, A\&AS, 61, 225

Andrei, A., Bouquillon, S., Camargo, S., et al. 2008, Proc. JSR2008, eds. M. Soffel, \& N. Capitaine, Lohrmann Observatorium and Observatoire de Paris

Andrei, A., Souchay, J., Zacharias, N., et al. 2009, A\&A, 505, 385

Anton, S., Andrei, A., \& Taris, F. 2012, Mem. Soc. Astron. It., 83, 3

Antonucci, R. 1993, ARA\&A, 31, 473

Arp, H. 1968, ApJ, 152, 1101

Bahcall, J., Kirhakos, S., \& Schneider, D. 1995, ApJ, 452, L91

Bertin, E., \& Arnouts, S. 1996, A\&AS, 117, 393

Bourda, G., Charlot, P., \& Le Campion, J. F. 2008, A\&A, 490, 403

Bourda, G., Charlot, P., Porcas, R., et al. 2010, A\&A, 520, A113

Boyce, P., Disney, M., \& Bleaken, D. 1999, MNRAS, 302, L39

Bozyan, E., Hemenway, D., \& Argue, A. 1990, AJ, 99, 142

Brindle, C. 1996, MNRAS, 282, 788

Carini, M., Walters, R., \& Hopper, L. 2011, AJ, 141, 49

Cheung, C., Wardle, J., \& Chen, T. 2003, New Astron. Rev., 47, 423

Coelho, B., Anton, S., Taris, F., et al. 2011, Proc. Journées Systèmes de Référence spatio-temporels

Costa, E. 2001, A\&A, 367, 719

Dai, B., Xie, G., Li, K., et al. 2001, AJ, 122, 2901

Damerdji, Y., Klotz, A., \& Boër, M. 2007, AJ, 133, 1470

Dolcini, A., Farfanelli, F., Ciprini, S., et al. 2007, A\&A, 469, 503

Dominici, T., Abraham, Z., Teixeira, R., et al. 2004, AJ, 128, 47

Falomo, R. 1996, MNRAS, 283, 241

Falomo, R., Urry, C., \& Pesce, J. 1997, ApJ, 476, 113

Falomo, R., Kotilainen, J., Pagani, C., et al. 2004, ApJ, 604, 495

Fan, J. 1997, Astro. Lett. Comm., 35, 361

Fan, J. H., \& Lin R. G. 2000a, ApJ, 537, 101

Fan, J. H., \& Lin R. G. 2000b, A\&A, 355, 880

Fan, J., Kurtanidze, O., Nikolashvili, M., et al. 2004, Chin. J. Astron. Astrophys., 4,133

Fey, A. L., \& Charlot, P. 1997, ApJS, 111, 95

Fey, A. L., Ma, C., Arias, E. F., et al. 2004, AJ, 127, 3587

Fey, A. L., Gordon, D. G., \& Jacobs, C. S. 2009, IERS Technical Note, 35 Fiorucci, M., \& Tosti, G., 1996, A\&AS, 117, 475

Francis, P., Whiting, M., \& Webster, R. 2000, PASA, 53, 56

Garcia, A., Sodre Jr, L., Jablonski, F., et al. 1999, MNRAS, 309, 803

Gaskell, C. 1982, ApJ, 252, 447

Gehren, T., Fried, J., Wehinger, P., et al. 1984, ApJ, 278, 11

Glass I. S. 2004, MNRAS, 350, 1049 
Gopal-Krishna, Stalin, C., \& Sagar, R. 2003, ApJ, 586, L25 Hawkins, M. 2002, MNRAS, 329, 76

Hawkins, M. 2003, MNRAS, 344, 492

Heidt, J., Troller, M., Nilsson, K., et al. 2004, A\&A, 418, 813

Jauncey, D., Wright, A., Peterson, B., et al. 1978, ApJ, 221, L109

Katajainen, S., Takalo, L., Sillanpaa, A., et al. 2000, A\&AS, 143, 357

Klotz, A., Boër, M., Eysseric, J., et al. 2008, PASP, 120, 1298

Kotilainen, J., Falomo, R., \& Scarpa, R. 1998a, A\&A, 332, 503

Kotilainen, J., Falomo, R., \& Scarpa, R. 1998b, A\&A, 336, 479

Laas-Bourez, M., Coward, D., Klotz., A., et al. 2010, AdSpR, 47, 402

Lasker, B., Lattanzi, M., McLean, B., et al. 2008, AJ, 136, 735

Lehnert, M., Miley, G., Sparks, W., et al. 1999, ApJS, 123, 351

Li, S., \& Cao, X. 2008, MNRAS, 387, L41

Lindegren, L. 2009, Proc. IAU Symp., 261, 296

Lobanov, A., \& Roland, J. 2005, A\&A, 431, 831

Ma, C., Arias, E. F., Eubanks, T. M., et al. 1998, AJ, 116, 516

Malkan, M. 1984, ApJ, 287, 555

Marquez, I., Petitjean, P., Théodore B., et al. 2001, A\&A, 371, 97

Miller, H. 1983, A\&AS, 52, 289

Moller, P., \& Warren, S. 1998, MNRAS, 299, 661

Nesci, R., Massaro, E., Rossi, C., et al. 2005, AJ, 130, 1466

Netzer, H., Heller, A., Loinger, F., et al. 1996, MNRAS, 279, 429

Nilsson, K., Charles, P., Pursino, T., et al. 1996, A\&A, 314, 754

Nilsson, K., Pursino, T., Heidt, J., et al. 2003, A\&A, 400, 95

Nilsson, K., Pursino, Sillanpaa, A., et al. 2008, A\&A, 487, L29

O'Dowd, M., \& Urry, C. 2005, ApJ, 627, 97

Orndahl, E., \& Ronnback, J. 2005, A\&A, 443, 61

Orndahl, E., Ronnback, J., \& Van Groningen, E. 2003, A\&A, 404, 883

Pica, A. J., Smith, A. G., Webb, J. R., et al. 1988, AJ, 96, 1215

Popovic, L., Jovanovic, P., Stalevski, M., et al. 2012, A\&A, 538, A107

Pursimo, T., Nilsson, K., Takalo, O., et al. 2002, A\&A, 381, 810
Raiteri, C., Villata, M., Tosti, G., et al. 1999, A\&A, 352, 19

Raiteri, C., Villata, M., \& Aller, H. 2001, A\&A, 377, 396

Raiteri, C., Villata, M., Kadler, M., et al. 2006, A\&A, 459, 731

Ronnback, J., Van Groningen, E., Wanders, I., et al. 1996, MNRAS, 283, 282

Sbarufatti, B., Treves, A., \& Falomo, R. 2005, ApJ, 635, 173

Sbarufetti, B., Falomo, R., Treves, A., et al. 2006, A\&A, 457, 35

Scarpa, R., Urry, C., Falomo, R., et al. 2000a, ApJ, 532, 740

Scarpa, R., Urry, C., Padovani, P., et al. 2000b, ApJ, 544, 258

Schramm, K., Borgeest, U., Kuhl, et al. 1994, A\&AS, 106, 349

Shalyapin, V., Goicoetchea, L., \& Alcalde, D. 2002, ApJ, 579, 127

Slavcheva-Mihova, L., \& Mihov, B. 2011, A\&A, 526, A43

Smith, A. G., \& Nair, A. D. 1995, PASP, 107, 863

Smith, A., Nair, A., Leacock, R., et al. 1993, AJ, 105, 437

Souchay, J., Andrei, A., Barache, C., et al. 2009, A\&A, 494, 799

Souchay, J., Andrei, A., Barache, C., et al. 2012, A\&A, 537, A99

Stalin, C., Gopal-Krishna, Sagar, R., \& Wiita, P. 2004, JApA, 25, 1

Stickel, M., Fried, J., \& Kuhr, H. 1993, A\&AS, 98, 393

Surace J. A., Sanders D. B., \& Evans, A. S. 2001, AJ, 122, 2791

Taris, F., Souchay, J., Andrei, A., et al. 2011, A\&A, 526, A25

Urry, C., \& Padovani, P. 1995, PASP, 107, 803

Urry, C., Scarpa, R., O’Dowd, M., et al. 2000, ApJ, 532, 816

Véron, P. 1996, A\&A, 310, 381

Véron-Cetty, M.-P., \& Véron, P. 2006, A\&A, 455, 773

Vigotti, M., Grueff, G., Perley, R., et al. 1989, AJ, 98, 419

Webb, J., Smith, A., Leacock, J., et al. 1988, AJ, 95, 374

Wright, S., Mc Hardy, I., Abraham, R., et al. 1998, MNRAS, 296, 961 Wurtz, R., Stocke, J., \& Yee, H. 1996, ApJ, 103, 109

Xie, G., Li, K., Cheng, F., et al. 1990, A\&A, 229, 329

Xie, G., Zhou, S., Dai, B., et al. 2002, MNRAS, 329, 689

Zackrisson, E., Bergvall, N., Marquart, T., et al. 2003, A\&A, 408, 17

Zekl, H., Klare, G., \& Appenzeller, I., 1981, A\&A, 103, 342 